\title{
Trophoblast glycoprotein promotes pancreatic ductal adenocarcinoma cell metastasis through Wnt/planar cell polarity signaling
}

\author{
PING HE $^{1 *}$, SHUHENG JIANG ${ }^{1,2^{*}}$, MINGZE MA ${ }^{1}$, YANG WANG ${ }^{1,2}$, \\ RONGKUN LI ${ }^{1}$, FANG FANG ${ }^{1}$, GUANGANG TIAN ${ }^{1}$ and ZHIGANG ZHANG ${ }^{1}$ \\ ${ }^{1}$ State Key Laboratory of Oncogenes and Related Genes, Shanghai Cancer Institute, Renji Hospital, \\ Shanghai Jiao Tong University School of Medicine, Shanghai 200240; ${ }^{2}$ Cancer Institute, \\ Shanghai Medical College of Fudan University, Shanghai 200032, P.R. China
}

Received May 16, 2014; Accepted February 9, 2015

DOI: $10.3892 / \mathrm{mmr} .2015 .3412$

\begin{abstract}
Trophoblast glycoprotein (TPBG), a 72 kDa glycoprotein was identified using a monoclonal antibody, which specifically binds human trophoblast. The expression of TPBG in normal tissues is limited; however, it is upregulated in numerous types of cancer. When TPBG is expressed at a high level, this usually indicates a poor clinical outcome. In the present study, it was demonstrated that TPBG was more commonly observed in human pancreatic ductal adenocarcinoma (PDAC) compared with normal pancreatic tissue. Immunohistochemical analysis of PDAC tissue microarrays indicated that the expression of TPBG in PDAC tissues was closely correlated with the tumor-node-metastasis stage of the tumor. Silencing of TPBG in PDAC cell lines resulted in a decreased ability of cancer cell migration and invasion. Further investigation demonstrated that the Wnt/planar cell polarity signaling pathway was suppressed, as the expression of Wnt5a and the activation of c-Jun N-terminal kinase was inhibited following TPBG knockdown. In conclusion, the present study provided evidence that TPBG is involved in PDAC metastasis, and that TPBG and its associated signaling pathways may be a suitable target for PDAC therapy.
\end{abstract}

Correspondence to: Dr Zhigang Zhang, State Key Laboratory of Oncogenes and Related Genes, Shanghai Cancer Institute, Renji Hospital, Shanghai Jiao Tong University School of Medicine, 800 Dongchuan Road, Shanghai 200240, P.R. China

E-mail: zzhang@shsci.org

*Contributed equally

Key words: Wnt/planar cell polarity signaling, invasion, trophoblast glycoprotein, pancreatic ductal adenocarcinoma, migration

\section{Introduction}

Pancreatic ductal adenocarcinoma (PDAC), usually referred to as pancreatic cancer, is a highly aggressive malignant tumor. It is the fourth leading cause of cancer-associated mortality with an estimated 37,390 fatalities in the USA and 227,000 fatalities globally per year (1). Patients with PDAC usually present with locally advanced, unresectable or metastatic disease and the majority of patients suffer significant pain (2). Despite the developments in the detection and management of PDAC, the five-year relative survival rate has not changed (3). Thus, further investigation into this malignant tumor is required.

Trophoblast glycoprotein (TPBG), also termed 5T4, is a $72 \mathrm{kDa}$ transmembrane glycoprotein and an extensively investigated oncofetal antigen, which is limited in normal adult tissues, but highly expressed in various types of cancer in humans $(4,5)$. This expression pattern renders it an attractive target for cancer therapy (6). Southall et al (5) observed that TPBG is highly expressed in tumor tissues, including carcinomas of the bladder, breast, ovaries, pancreas and stomach, as elucidated using immunohistochemical analysis, and was closely associated with poor clinical outcome in colorectal, ovarian and gastric cancer (7-9). The authors further demonstrated that TPBG not only disrupts cell-cell contacts and induces cellular motility in epithelial cells (10), but also interacts with GAIP-interacting protein $\mathrm{C}$ terminus 1 , which has implications for tumor metastasis (11).

However, the roles of TPBG in PDAC progression remain to be elucidated. The present study aimed to explore the cellular functions of TPBG in PDAC, and to investigate the associated mechanisms.

\section{Materials and methods}

Immunohistochemical staining. The PDAC tissue microarray (OD-CT-DgPan01-006), which contains 81 PDAC tissues, 44 normal pancreatic tissues and 32 chronic pancreatitis tissues was purchased from Shanghai Outdo Biotech Co., Ltd. (Shanghai, China). The immunohistochemical staining was performed as previously described (12). Briefly, tissue 
sections were deparaffinized gradually using 50\% xylene (Meryer, Shanghai, China) and rehydrated. The sections were then incubated with $0.3 \%$ hydrogen peroxide (Jianglaibio, Shanghai, China) for $30 \mathrm{~min}$ and blocked with $10 \%$ bovine serum albumin (BSA; Sangon, Shanghai, China). The slides were initially incubated with an antibody targeting TPBG (cat. no. HPA010554; Sigma-Aldrich, St. Louis, MO, USA) at $4^{\circ} \mathrm{C}$ overnight with optimal dilution (1:200). The slides were then incubated with a horsradish peroxidase-conjugated rabbit secondary antibody (cat. no. 65-6120; Thermo Fisher Scientific, Waltham, MA, USA) at room temperature for 1 hour. Subsequently, the slides were incubated with diaminobenizidine substrate liquid (Gene Tech Ltd., Shanghai, China), for $2 \mathrm{~min}$ and counterstained with hematoxylin (Beyotime Institue of Biotechnology, Haimen, China). Then these sections were ehydrated sequentially using 50, 80, 90 and $100 \%$ ethanol (Meryer), and 50 and $100 \%$ xylene, and covered. The slides were subsequently visualized using Primostar FL2 microscope (CarlZeiss, Oberkochen, Germany).

Cell culture. The PANC-1 and BxPC-3 human PDAC cell lines were obtained from the Cell Bank of the Chinese Academy of Sciences (Shanghai, China), and the hTERT-HPNE normal pancreatic duct cell line was purchased from the American Type Culture Collection (ATCC; Manassas, VA, USA). PANC-1 cells were cultured with Dulbecco's modified Eagle's medium (Beijing Solarbio Science \& Technology Co., Ltd., Beijing, China), BxPC-3 cells were cultured with RMPI 1640 (Beijing Solarbio Science \& Technology Co., Ltd.) and hTERT-HPNE cells were cultured with specific medium recommended by the ATCC [75\% glucose-free Dulbecco's modified Eagle's medium (Gibco Life Technologies, Carlsbad, CA, USA), 25\% Medium M3 Base (Gibco Life Technologies)] supplemented with $5 \%$ fetal bovine serum (FBS), $10 \mathrm{ng} / \mathrm{ml}$ human recombinant epidermal growth factor (PeproTech, New Jersey, NJ, USA), $5.5 \mathrm{mM}$ D-glucose (Gibco Life Technologies) and $750 \mathrm{ng} / \mathrm{ml}$ puromycin (Sangon, Shanghai, China)]. All cells were supplemented with $10 \%$ FBS (v/v; Gibco Life Technologies, Carlsbad, CA, USA), 100 units penicillin (Gibco Life Technologies) and $100 \mu \mathrm{g}$ streptomycin (Gibco Life Technologies) and incubated at $37^{\circ} \mathrm{C}$ in a humidified incubator with $5 \% \mathrm{CO}_{2}$.

Construction of stably interfered TPBG cell lines. Short hairpin (sh)RNAs and control vector were purchased from GenePharma (Shanghai, China). The packaging of lentiviral particles was performed in HEK293T cells (ATCC, Manassas, VA, USA)following cotransfection with shRNAs with the following sequences: sh-1, 5'-CCGGGGA TCACATGGAAGGGTATCACTCGAGTGATACCCTTCCA TGTGATCCTTTTTG-3' and sh-2, 5'-CCGGGCACAGT CAAGTGCGTTAACCCTCGAGGGTTAACGCACTTGAC

TGTGCTTTTTG-3'. Control vectors were transfected using Lipofectamine 2000 (Invitrogen Life Technologies, Carlsbad, CA, USA). The viruses were harvested at 24 and $48 \mathrm{~h}$ after transfection. The PANC-1 and BxPC-3 cells $\left(1 \times 10^{5}\right)$ were seeded into 12-well plates and infected with lentivirus in the presence of $6 \mu \mathrm{g} / \mathrm{ml}$ polybrene (Sigma-Aldrich) on the following day. The infected PANC-1 and BxPC-3 cells were then selected with medium containing $2 \mu \mathrm{g} / \mathrm{ml}$ puromycin (Sangon Biotech) for 7 days. The puromycin resistant cells were considered to be cells that had undergone stable knockdown of TPBG as verified using reverse transcription-quantitative polymerase chain reaction (RT-qPCR) and western blot analysis.

$R T$-qPCR. Total RNA was extracted using TRIzol reagent (Takara Bio Inc., Otsu, Japan) and reverse transcribed using the PrimeScript RT-PCR kit (Takara Bio Inc.) according to the manufacturer's instructions. RT-qPCR analysis was performed using SYBR Premix Ex Taq (Takara Bio Inc.) with the ViiA 7 Real-time PCR system (Applied Biosystems, Foster City, CA, USA), with the following program: $95^{\circ} \mathrm{C}$ for $30 \mathrm{sec}$, followed by 40 cycles at $95^{\circ} \mathrm{C}$ for $5 \mathrm{sec}$ and $60^{\circ} \mathrm{C}$ for $31 \mathrm{sec}$, and finally $95^{\circ} \mathrm{C}$ for $15 \mathrm{sec}, 60^{\circ} \mathrm{C}$ for $1 \mathrm{~min}$ an $\mathrm{d} 95^{\circ} \mathrm{C}$ for $15 \mathrm{sec}$. The primer sequences for TPBG and 18S RNA in the present study were as follows: forward: 5'-TGGGTATTGTTTTAGCCCTGAT-3' and reverse: 5'-GTTGTCCTTGGTCTGTCCTCTA-3' for TPBG, and forward: 5'-TGCGAGTACTCAACACCAACA-3' and reverse: 5'-GCATATCTTCGGCCCACA-3' for 18S RNA. The relative expression of TPBG was calculated using the $2^{-\Delta \Delta C T}$ method with $18 \mathrm{~S}$ RNA as the reference gene.

Western blotting. Total proteins were extracted using an immunoprecipitation lysis buffer (P0013; Beyotime Institute of Biotechnology, Haimen, China) according to the manufacturer's instructions and proteins were then separated using reducing SDS-PAGE and transferred onto a nitrocellulose membrane (GE Healthcare Life Sciences, Pittsburgh, PA, USA). Subsequently, the membrane was blocked in Tris-buffered saline (Beijing Solarbio Science \& Technology Co.) containing 5\% BSA for $1 \mathrm{~h}$. The membrane was incubated with primary antibodies against TPBG (1:500; cat. no. HPA010554; Sigma-Aldrich), Wnt5a (1:1,000; cat. no. 2392; Cell Signaling Technology, Danvers, MA, USA), ROR2 (1:1,000; cat. no. 4105; Cell Signaling Technology) c-Jun N-terminal kinase (JNK; 1:1,000; cat. no. 9252; Cell Signaling Technology), phospho-JNK (1:1,000; cat. no. 4668; Cell Signaling Technology) and $\beta$-actin (1:5,000; cat. no. 20536-1-AP; Proteintech, Chicago, IL, USA) overnight and then the membranes were incubated with species-specific secondary antibodies, IRDye 680 anti-mouse (1:20,000; cat. no. 926-68072; LI-COR, Lincoln, NE, USA) or IRDye 800 anti-rabbit (1:10,000; cat. no. 926-32213L; LI-COR) to probe the primary antibodies while signals were detected using the Odyssey infrared imaging system (LI-COR) as described previously (13).

In vitro cell migration and invasion assays. Transwell migration and invasion assays were performed. For the cell migration assay, $4 \times 10^{4}$ cells were seeded into the upper compartment of the Transwell inserts (Millipore, Billerica, MA, USA) and the lower compartment was supplemented with $700 \mu$ l conditioned medium containing 5\% FBS (v/v). The cells remaining in the top chambers or on the upper membrane of the inserts were carefully removed after incubation for $10 \mathrm{~h}$. The cells that migrated to the lower membrane of the inserts were fixed with $2 \%$ glutaraldehyde and stained with $0.1 \%$ crystal violet (Beyotime Institute of Biotechnology). Subsequently, migrated cells were counted by capturing images of six randomly selected fields through an IX71 inverted microscope (Olympus Corp., Tokyo, Japan). A cell invasion assay was performed by adding $100 \mu \mathrm{l}$ Matrigel (BD Biosciences, Mountain View, 

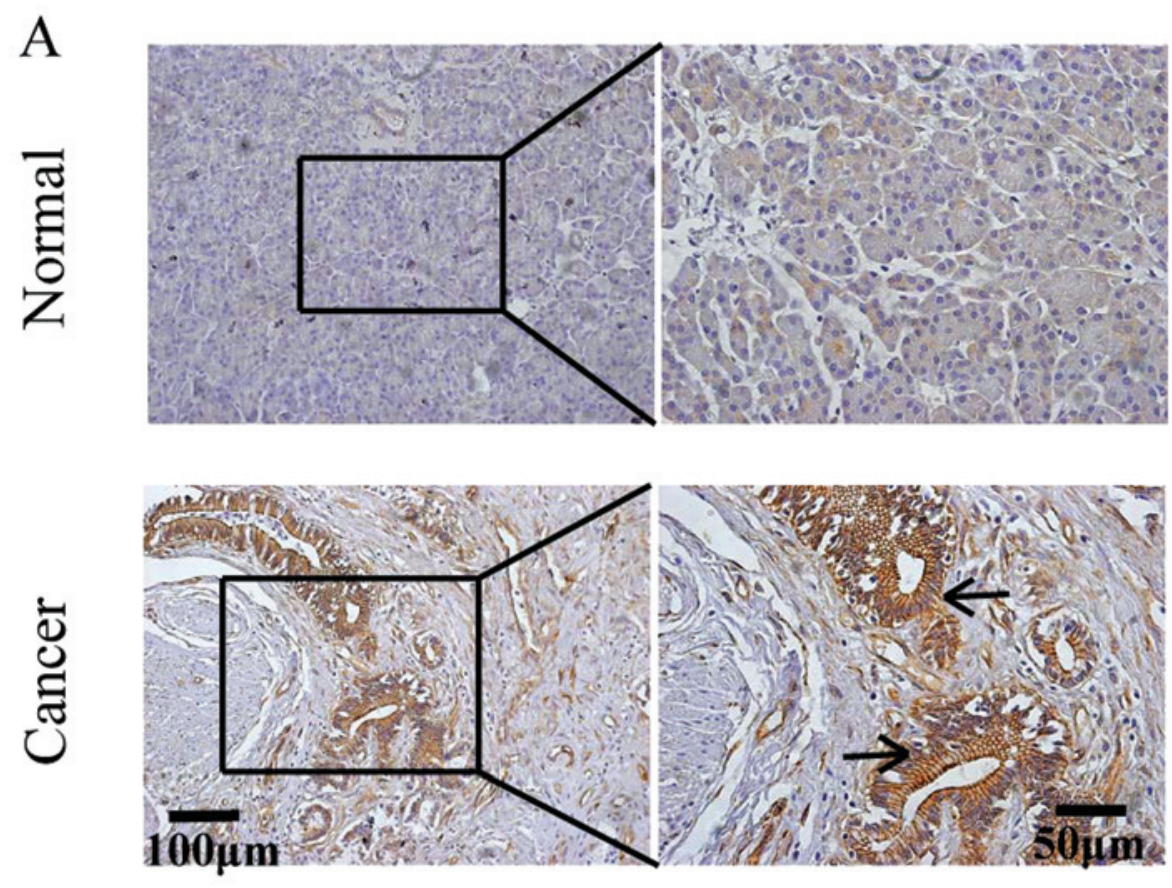

B

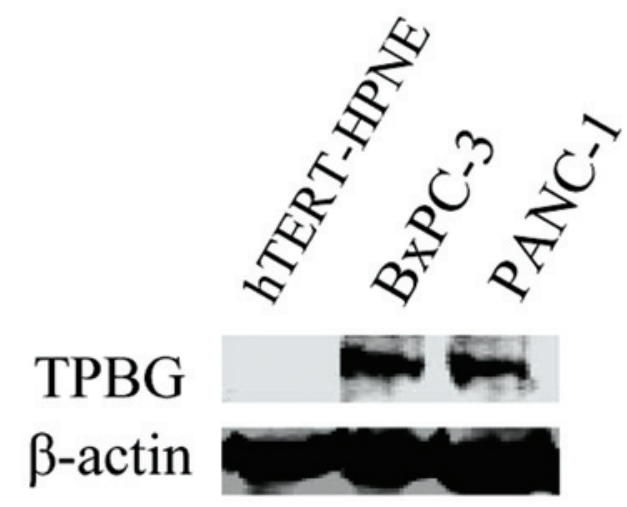

Figure 1. TPBG is highly expressed in PDAC tissues and cell lines. (A) Representative images of TPBG immunoreactivity in pancreatic carcinoma cells and normal control cells. Arrows indicate TPBG positive staining in pancreatic carcinoma. (B) TPBG levels were detected in BxPC-3 and PANC-1 PDAC cell lines, and the hTERT-HPNE normal pancreatic duct cell line using western blotting. TPBG, trophoblast glycoprotein; PDAC, pancreatic ductal adenocarcinoma.

CA, USA) into the top chamber of the Transwell inserts and placing $4 \times 10^{4}$ primary cells onto the Matrigel. The cell invasion assay was allowed to continue for 14-20 $\mathrm{h}$ and was then terminated and treated according to the cell migration assay described above.

Luciferase reporter assay. Cells were seeded into the 96-well plates and transfected with a mixture of $100 \mathrm{ng}$ ATF2 plasmid (Wnt/PCP signaling) and $10 \mathrm{ng}$ Renilla plasmid according to the manufacturer's instructions of the Lipofectamine 2000 transfection system. After $48 \mathrm{~h}$ of incubation, firefly and Renilla luciferase activity of cell lysates was measured using the dual-luciferase reporter assay system (Promega Corporation, Madison, WI, USA).

Statistical analysis. Data are expressed as the mean \pm standard deviation. The correlation between TPBG expression and the clinicopathological parameters was evaluated using the $\chi^{2}$ test. Student's t-test was used for comparisons between groups. $\mathrm{P}<0.05$ was considered to indicate a statistically significant difference.

\section{Results}

TPBG is highly expressed in PDAC tissues and cell lines. To observe the change in expression occurring in PDAC, the expression of TPBG was initially identified in a tissue microarray, which contained 81 cases of PDAC. Through immunohistochemical staining, it was revealed that TPBG is highly expressed in cancer tissues compared with the normal pancreatic tissues (Fig. 1). In addition, a high expression of TPBG was closely correlated with tumor-node-metastasis (TNM) stage and the age of the patient (Table I). In addition, the expression of TPBG was detected in the hTERT-HPNE normal pancreatic duct cell line and the PANC-1 and BxPC-3 PDAC cell lines. The expression of TPBG was detected at 
Table I. Statistical analysis of TPBG expression against clinicopathological parameters of the patient.

\begin{tabular}{|c|c|c|c|}
\hline \multirow[b]{2}{*}{ Variable } & \multicolumn{2}{|c|}{ TPBG, n (\%) } & \multirow[b]{2}{*}{ P-value } \\
\hline & High & Low & \\
\hline \multicolumn{4}{|l|}{ Sample } \\
\hline Carcinoma & $49(60.49)$ & $32(39.51)$ & \\
\hline Normal & $10(22.73)$ & $34(77.27)$ & \\
\hline Pancreatitis & $19(59.38)$ & $13(40.62)$ & 0.913 \\
\hline Age (years) & & & 0.022 \\
\hline$\leq 60$ & $28(73.68)$ & $10(26.32)$ & \\
\hline$>60$ & $21(48.84)$ & $22(51.16)$ & \\
\hline Gender & & & 0.062 \\
\hline Female & $24(72.73)$ & $9(27.27)$ & \\
\hline Male & $25(52.08)$ & $23(47.91)$ & \\
\hline Tumor size $(\mathrm{cm})$ & & & 0.051 \\
\hline$\leq 4$ & $14(46.67)$ & $16(53.33)$ & \\
\hline$>4$ & $35(68.63)$ & $16(31.37)$ & \\
\hline Tumor location & & & 0.45 \\
\hline Pancreas head & $36(84.00)$ & $21(16.00)$ & \\
\hline Pancreas body and tail & $13(54.17)$ & $11(45.83)$ & \\
\hline TNM stage & & & 0.005 \\
\hline I & $18(45.00)$ & $22(55.00)$ & \\
\hline II-III & $31(75.61)$ & $10(24.39)$ & \\
\hline
\end{tabular}

Data in bold indicate P-values with significant differences. TPBG, trophoblast glycoprotein; TNM, tumor-node-metastasis.

A

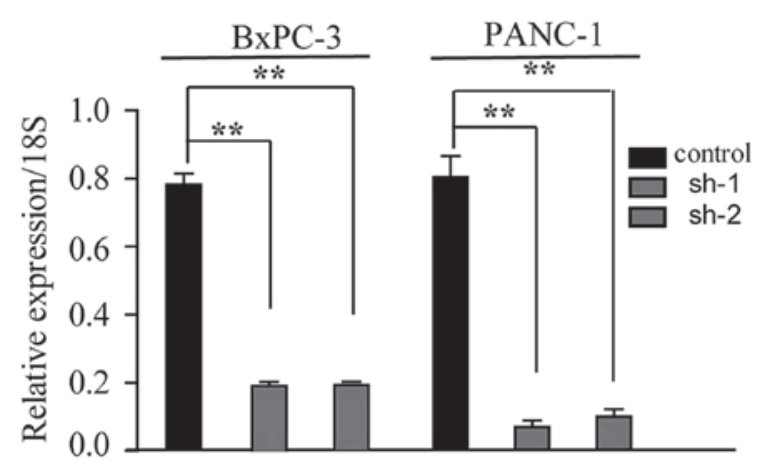

B BxPC-3

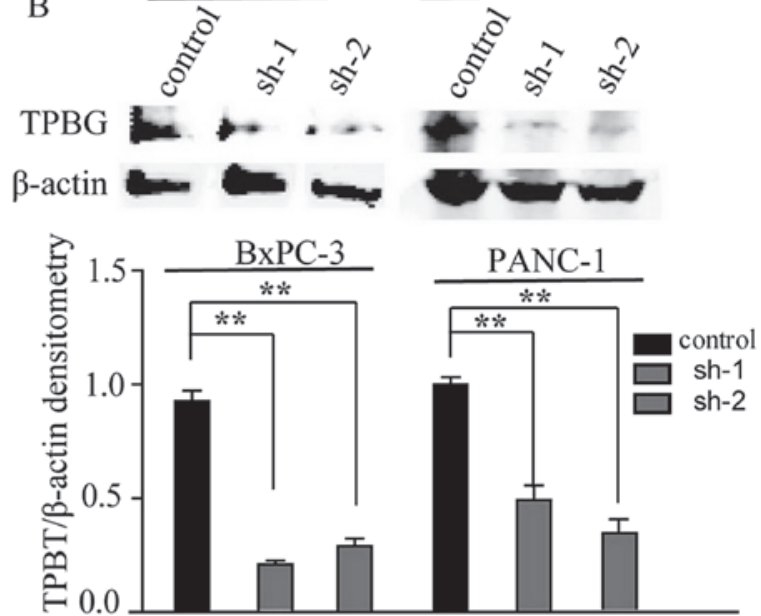

Figure 2. Expression of TPBG decreased significantly in cell lines with stable silencing. (A) Expression level of TPBG was detected by reverse transcription-quantitative polymerase chain reaction. (B) Expression level of TPBG was detected by western blotting in BxPC-3 and PANC-1 cells following TPBG knockdown, the densitometric value was normalized to the internal control and relative expression with the following equation: Normalization of the two groups/the first normalized value. Data are expressed as the mean \pm standard deviation, $n=3 ;{ }^{* *} \mathrm{P}<0.01$. TPBG, trophoblast glycoprotein; sh, short hairpin $\mathrm{RNA}$.

extremely low levels in hTERT-HPNE, while it was highly expressed in the PANC-1 and BxPC-3 cell lines (Fig. 1B).

Expression of TPBG in BxPC-3 and PANC-1 cell lines is significantly decreased following stable silencing. To further investigate the role of TPBG in PDAC carcinogenesis and progression, the BxPC-3 and PANC-1 PDAC cell lines were selected, which highly expressed TPBG, to construct cell lines exhibiting stable knockdown of TPBG. The silencing of TPBG by two shRNAs (sh-1 and sh-2) in the BxPC-3 and PANC-1 
A
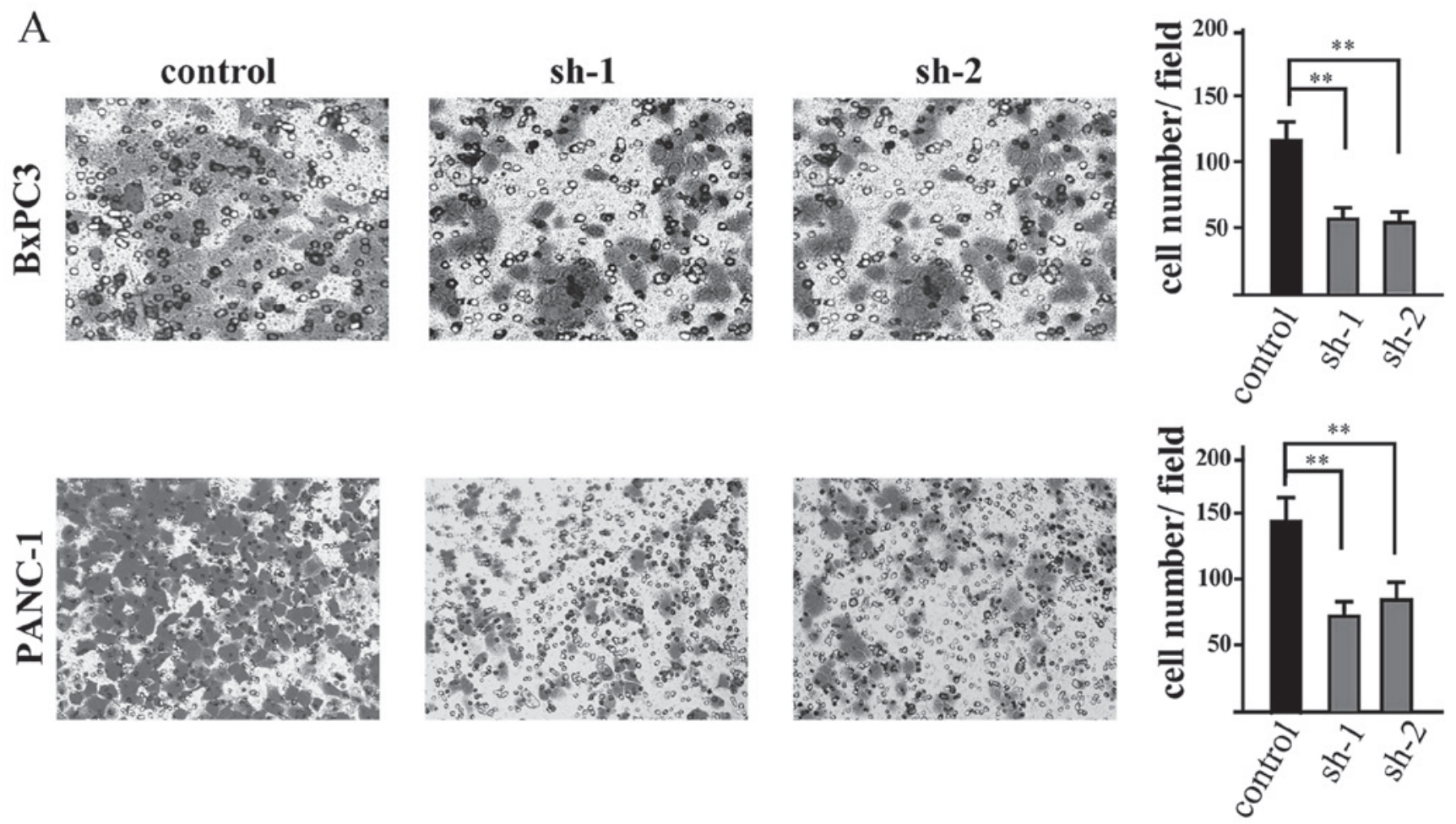

B
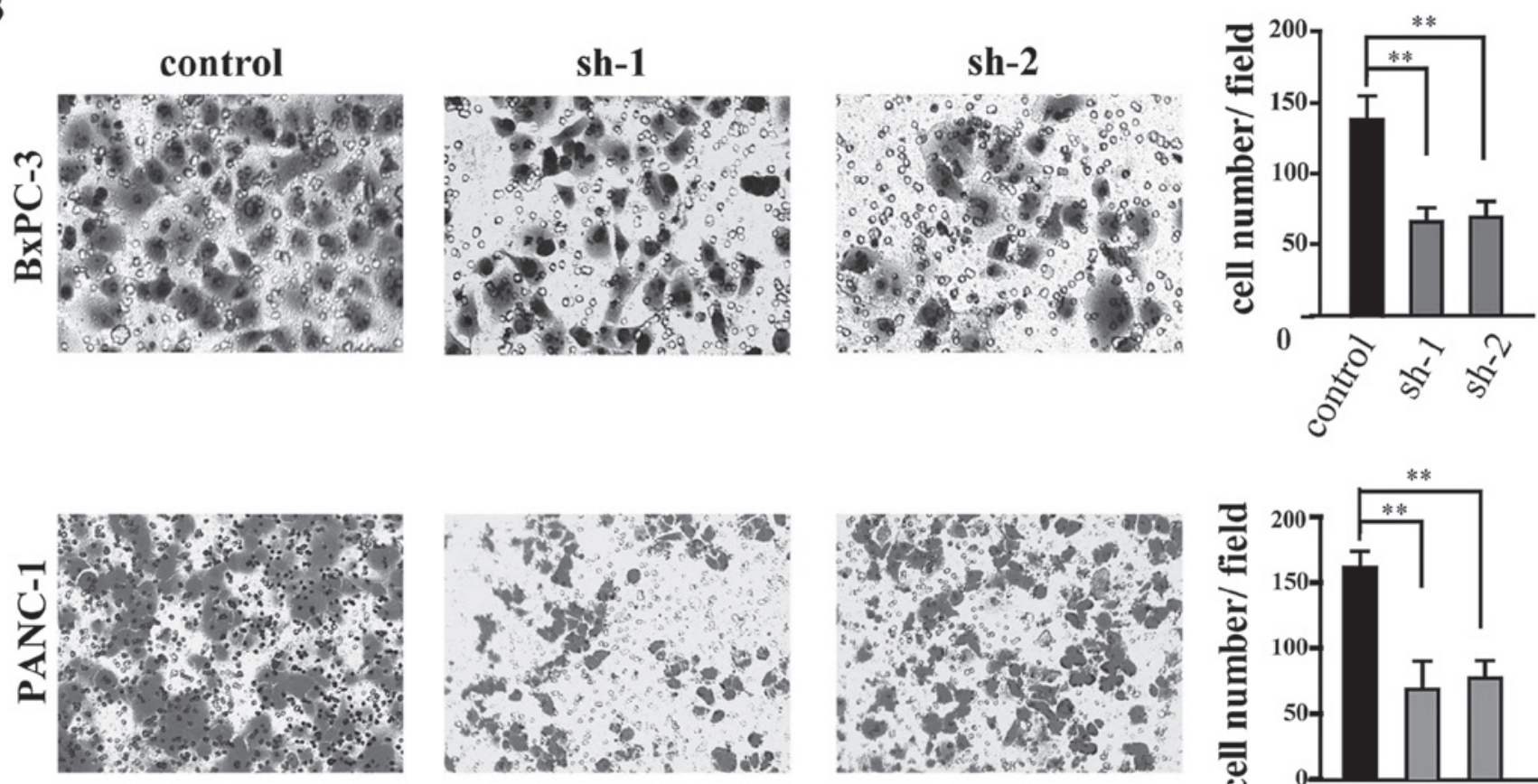

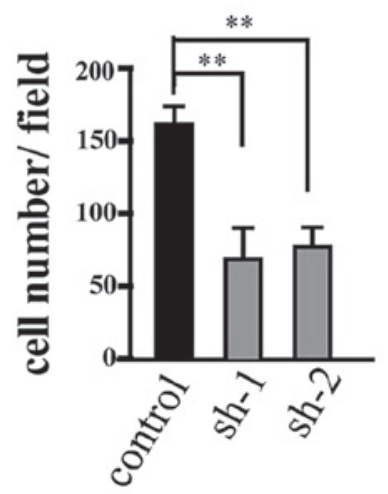

Figure 3. Silencing of TPBG reduces the ability of PDAC cell migration and invasion. (A) Representative images of BxPC-3 and PANC-1 cells migrated to the lower compartment following TPBG knockdown and quantification analysis (right panel). (B) Representative images of BxPC-3 and PANC-1 cells invaded to the lower compartment following TPBG knockdown and quantification analysis (right panel). Data are expressed as the mean \pm standard deviation, $\mathrm{n}=3$; ${ }^{* *} \mathrm{P}<0.01$. TPBG, trophoblast glycoprotein; PDAC, pancreatic ductal adenocarcinoma; sh, short hairpin RNA.

cells resulted in significant decreases in the expression of TPBG (Fig. 2).

Silencing of TPBG inhibits PDAC cell migration and invasion. Given that TPBG is a gene that is associated with cell motility, a Transwell assay was performed. In the migration assay, it was observed that the motility of the cells was notably decreased following TPBG knockdown (Fig. 3A). In addition, to examine whether TPBG has an affect on the invasive ability of the cells, a cell invasion assay was performed and it was identified that 

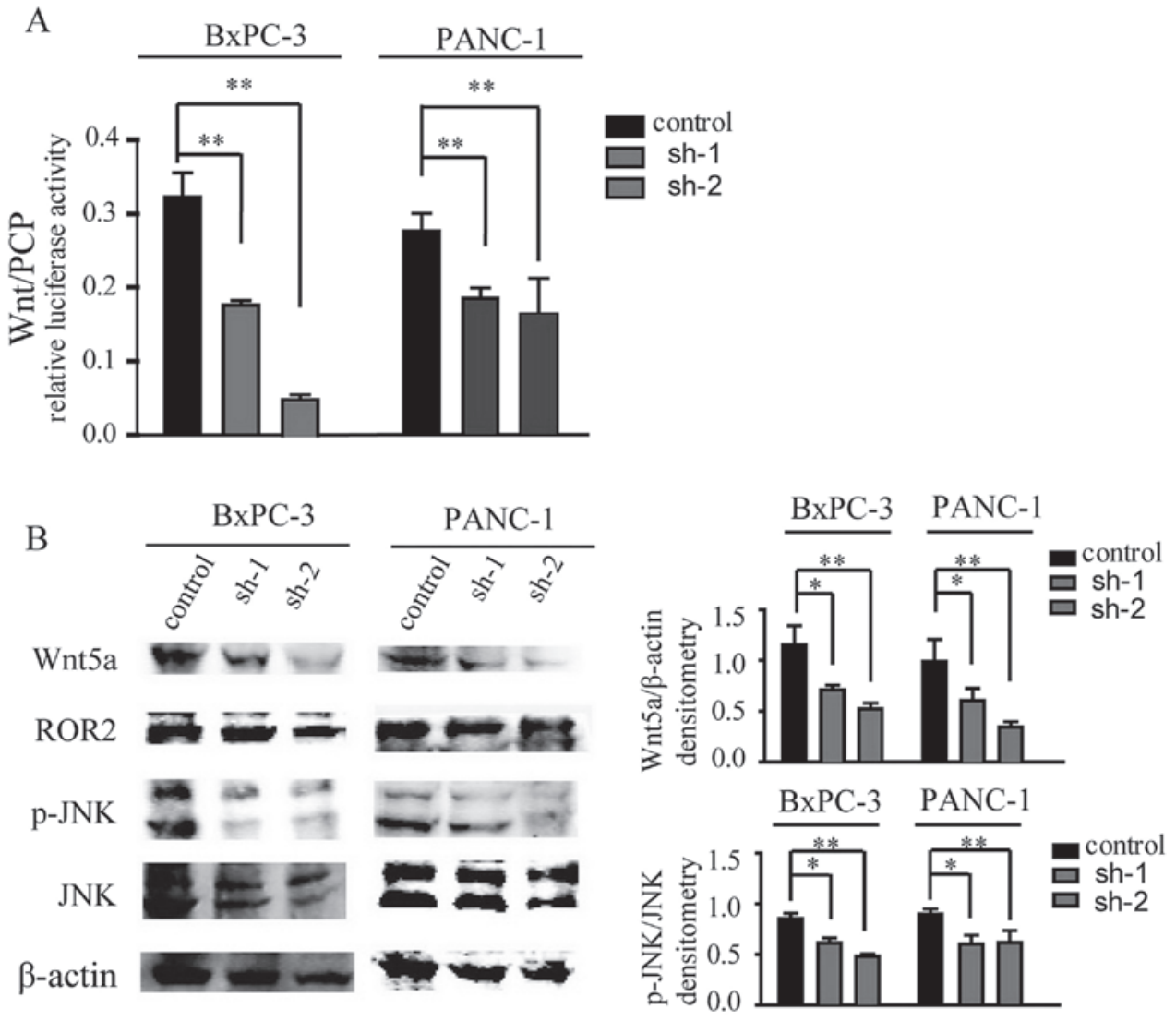

Figure 4. Wnt/PCP signaling is inhibited following TPBG knockdown in PDAC cell lines. (A) Dual-luciferase reporter assay demonstrated that Wnt/PCP signaling was inhibited significantly in BxPC-3 and PANC-1 cells following TPBG knockdown. (B) Expression of Wnt5a and the activation of JNK were decreased following TPBG knockdown, $\beta$-actin was used as a loading control. The densitometric value was normalized to the internal control and relative expression with the following equation: Normalization of the two groups/the first normalized value. Data are expressed as the mean \pm standard deviation, $\mathrm{n}=3$; ${ }^{*} \mathrm{P}<0.05$ and ${ }^{* *} \mathrm{P}<0.01$. TPBG, trophoblast glycoprotein; PDAC, pancreatic ductal adenocarcinoma; JNK, c-Jun N-terminal kinase; PCP, planar cell polarity.

number of cells that invaded through the membrane was also decreased significantly following silencing of TPBG (Fig. 3B).

Wnt/PCP pathway is suppressed following silencing of TPBG. To further investigate the underlying mechanism involved in cell migration and invasion in $\mathrm{PDAC}, \mathrm{Wnt} / \mathrm{PCP}$ signaling was examined, which has been observed to promote cell migration in cancer metastasis (14). Using a luciferase reporter assay, it was identified that Wnt/PCP signaling was inhibited in the cells subjected to TPBG knockdown compared with the control cells (Fig. 4A). Collectively, the present results suggested that TPBG may affect cell migration and invasion through the Wnt/PCP signaling pathway. To further confirm these results, several molecules associated with the Wnt/PCP pathway were investigated. It was identified that the expression of Wnt5a, an extensively investigated Wnt ligand of the Wnt/PCP pathway $(15,16)$ was inhibited (Fig. 4B). The activation of JNK, a downstream molecule of the Wnt/PCP pathway, was also reduced markedly (Fig. 4B). Thus, the present data indicated that TPBG may affect cell migration and invasion through the Wnt/PCP pathway.

\section{Discussion}

In the present study, the aberrant expression of TPBG in PDAC was described and the potential effect of highly expressed
TPBG in PDAC cell lines was investigated. In previous studies, immunohistochemical analysis has revealed that TPBG is overexpressed in numerous types of cancer (5). In addition, the expression of TPBG usually indicates a poor prognosis, with TPBG expression correlating with the likelihood of cancer metastasis $(7-9,17)$. Consistent with these observations, the present results provide evidence that TPBG may be a critical factor that facilitates PDAC progression and metastasis. In the present study, immunohistochemical analysis of a PDAC tissue microarray revealed that TPBG is closely associated with the TNM stage of the tumor. The cell migration and invasion abilities were significantly decreased following TPBG knockdown. TPBG expression is, therefore, correlated with the aggressiveness of PDAC, including the metastatic properties of the malignancy.

However, how TPBG regulates PDAC cell migration remains to be fully elucidated. The Wnt/PCP signaling pathway has been revealed to be involved in cell movement through the activation of the RhoA, JNK and nemo-like kinase signaling cascades (18). In addition, accumulating evidence has suggested that Wnt/PCP signaling is closely associated with tumors and has diverse roles in tumorigenesis $(18,19)$. Previous studies have demonstrated that TPBG activates the Wnt/PCP pathways by modifying the subcellular localization of low density lipoprotein receptor-related protein 6 during Zebrafish development (20). These findings imply that TPBG may affect 
Wnt/PCP signaling in PDAC, thus the correlation between TPBG and Wnt/PCP signaling was further investigated.

It was identified that Wnt/PCP signaling was inhibited significantly in the PDAC cell lines following TPBG knockdown via a luciferase reporter assay. Numerous studies have suggested that Wnt5a, a typical noncanonical Wnt ligand, promotes the metastasis of melanoma, gastric cancer and breast cancer by activating the Rac and JNK signaling pathways $(14,21,22)$. The expression of Wnt5a, Ror2 (a receptor for Wnt5a) (23) and a change in phospho-JNK were also detected and it was observed that the expression of Wnt5a and the activation of phospho-JNK were decreased markedly in the cell lines in which TPBG expression was silenced. Collectively, the present results demonstrated that TPBG promotes cancer metastasis partly through Wnt/PCP signaling. However, numerous factors remain to be elucidated, including how TPBG regulates the expression of Wnt5a and how TPBG affects the downstream molecules of the Wnt/PCP pathway.

In conclusion, the present study demonstrated that TPBG expression is upregulated in human PDAC tissues and the highly expressed TPBG may regulate the Wnt/PCP signaling to enhance the abilities of cancer cell migration and invasion. The findings of the present study indicate that the distinctive expression pattern of TPBG may render it a suitable target for the treatment of PDAC.

\section{Acknowledgements}

The present study was supported by the National High Technology Research and Development Program of China (863 Program; grant no. 2014AA020609) and the State Key Laboratory Project (grant no. 91-14-08, 91-14-14).

\section{References}

1. Vincent A, Herman J, Schulick R, Hruban RH and Goggins M: Pancreatic cancer. Lancet 378: 607-620, 2011.

2. Helm JF, Centeno BA, Coppola D, et al: Outcomes following resection of pancreatic adenocarcinoma: 20-year experience at a single institution. Cancer Control 15: 288-294, 2008.

3. Siegel R, Naishadham D and Jemal A: Cancer statistics, 2013. CA Cancer J Clin 63: 11-30, 2013.

4. Hole N and Stern PL: A $72 \mathrm{kD}$ trophoblast glycoprotein defined by a monoclonal antibody. Br J Cancer 57: 239-246, 1988.

5. Southall PJ, Boxer GM, Bagshawe KD, Hole N, Bromley M and Stern PL: Immunohistological distribution of 5T4 antigen in normal and malignant tissues. Br J Cancer 61: 89-95, 1990.
6. Harrop R, Drury N, Shingler W, et al: Vaccination of colorectal cancer patients with modified vaccinia ankara encoding the tumor antigen 5T4 (TroVax) given alongside chemotherapy induces potent immune responses. Clin Cancer Res 13: 4487-4494, 2007.

7. Starzynska T, Marsh PJ, Schofield PF, Roberts SA, Myers KA and Stern PL: Prognostic significance of 5T4 oncofetal antigen expression in colorectal carcinoma. Br J Cancer 69: 899-902, 1994.

8. Wrigley E, McGown AT, Rennison J, et al: $5 \mathrm{~T} 4$ oncofetal antigen expression in ovarian carcinoma. Int $\mathrm{J}$ Gynecol Cancer 5: 269-274, 1995.

9. Naganuma H, Kono K, Mori Y, et al: Oncofetal antigen 5T4 expression as a prognostic factor in patients with gastric cancer. Anticancer Res 22: 1033-1038, 2002.

10. Carsberg CJ, Myers KA and Stern PL: Metastasis-associated 5T4 antigen disrupts cell-cell contacts and induces cellular motility in epithelial cells. Int J Cancer 68: 84-92, 1996.

11. Awan A, Lucic MR, Shaw DM, et al: 5T4 interacts with TIP-2/GIPC, a PDZ protein, with implications for metastasis. Biochem Biophys Res Commun 290: 1030-1036, 2002.

12. Ma M-Z, Zhuang C, Yang X-M, et al: CTHRC1 acts as a prognostic factor and promotes invasiveness of gastrointestinal stromal tumors by activating Wnt/PCP-Rho signaling. Neoplasia 16: 265-278, 2014

13. Li J, Yang X-M, Wang Y-H, et al: Monoamine oxidase a suppresses hepatocellular carcinoma metastasis by inhibiting the adrenergic system and its transactivation of EGFR signaling. J Hepatol 60: 1225-1234, 2014.

14. Weeraratna AT, Jiang Y, Hostetter G, et al: Wnt5a signaling directly affects cell motility and invasion of metastatic melanoma. Cancer Cell 1: 279-288, 2002.

15. Yamamoto H, Oue N, Sato A, et al: Wnt5a signaling is involved in the aggressiveness of prostate cancer and expression of metalloproteinase. Oncogene 29: 2036-2046, 2010.

16. Vuga LJ, Ben-Yehudah A, Kovkarova-Naumovski E, et al: WNT5A is a regulator of fibroblast proliferation and resistance to apoptosis. Am J Respir Cell Mol Biol 41: 583-589, 2009.

17. Castro FV, McGinn OJ, Krishnan S, et al: 5T4 oncofetal antigen is expressed in high risk of relapse childhood pre-B acute lymphoblastic leukemia and is associated with a more invasive and chemotactic phenotype. Leukemia 26: 1487-1498, 2012.

18. Katoh M: WNT/PCP signaling pathway and human cancer (review). Oncol Rep 14: 1583-1588, 2005.

19. Wang Y: Wnt/Planar cell polarity signaling: a new paradigm for cancer therapy. Mol Cancer Ther 8: 2103-2109, 2009.

20. Kagermeier-Schenk B, Wehner D, Özhan-Kizil G, et al: Waif1/5T4 inhibits Wnt/ $\beta$-catenin signaling and activates noncanonical Wnt pathways by modifying LRP6 subcellular localization. Dev Cell 21: 1129-1143, 2011.

21. Kurayoshi M, Oue N, Yamamoto H, et al: Expression of Wnt-5a is correlated with aggressiveness of gastric cancer by stimulating cell migration and invasion. Cancer Res 66: 10439-10448, 2006.

22. Pukrop T, Klemm F, Hagemann T, et al: Wnt 5a signaling is critical for macrophage-induced invasion of breast cancer cell lines. Proc Natl Acad Sci USA 103: 5454-5459, 2006.

23. Oishi I, Suzuki H, Onishi N, et al: The receptor tyrosine kinase Ror2 is involved in non-canonical Wnt5a/JNK signalling pathway. Genes Cells 8: 645-654, 2003. 